\title{
A solar cycle lengthwise series of solar diameter measurements
}

\author{
J. L. Penna ${ }^{1}$, A. H. Andrei ${ }^{1,2, *}$, S. C. Boscardin ${ }^{1}$, E. Reis Neto ${ }^{1}$ \\ and V. A. d'Ávila ${ }^{1,3}$ \\ ${ }^{1}$ Observatório Nacional/MCT, R. Gal. Jose Cristino 77, Rio de Janeiro, Brazil \\ email(*): oat1@on.br \\ ${ }^{2}$ Obs. do Valongo/UFRJ-BR, Rio de Janeiro, Brazil \\ ${ }^{3}$ UERJ-BR, Rio de Janeiro, Brazil
}

\begin{abstract}
The measurements of the solar photospheric diameter rank among the most difficult astronomic observations. Reasons for this are the fuzzy definition of the limb, the SNR excess, and the adverse daytime seeing condition. As a consequence there are very few lengthy and consistent time series of such measurements. Using modern techniques, just the series from the IAG/USP and from Calern/OCA span more than one solar cycle. The Rio de Janeiro Group observations started in 1997, and therefore in 2008 one complete solar cycle time span can be analyzed. The series shares common principles of observation and analysis with the ones afore mentioned, and it is complementary on time to them. The distinctive features are the larger number of individual points and the improved precision. The series contains about 25,000 single observations, evenly distributed on a day-by-day basis. The typical error of a single observation is half an arc-second, enabling us to investigate variations at the expected level of tens of arc- second on a weekly basis. These features prompted to develop a new methodology for the investigation of the heliophysical scenarios leading to the observed variations, both on time and on heliolatitude. The algorithms rely on running averages and time shifts to derive the correlation and statistical incertitude for the comparison of the long term and major episodes variations of the solar diameter against activity markers. The results bring support to the correlation between the diameter variation and the solar activity, but evidentiating two different regimens for the long term trend and the major solar events.
\end{abstract}

Keywords. Sun: photosphere, fundamental parameters, activity; instrumentation: photometers; methods: statistical

\section{Introduction}

The bulk of solar light and heat received by the Earth is released from the solar photosphere. Though virtually all the energy is produced in the solar core through the proton-proton fusion chain, it undergoes a lengthy pathway, across the radiation zone and up to the convective zone, during a million years process, to finally reach what is purposely called the solar photosphere (Mursula, 2005).

The solar constant $(\mathbf{W})$ expresses the total irradiance, i.e., the total power of solar radiation over the full frequency spectrum per perpendicular area at the mean distance of the Earth. The solar constant is hardly constant though. It varies by a factor of

(a) $10^{-6}$ over minutes. (These are related to pressure variations called solar oscillations),

(b) $10^{-3}$ over several days. (These are related to sunspots and other active regions),

(c) $10^{-3}$ over solar cycle (exact value uncertain).

The solar energy is one of the major driving inputs for terrestrial climate. Some evidences of correlations exist between surface temperature changes and solar activity. It is 
then important to know on what time scale the solar irradiance and other fundamental solar parameters, like the diameter, vary in order to better understand and assess the origin and mechanisms of the terrestrial climate changes.

Global effects, such as diameter changes, large convective cells, the differential rotation of the Sun's interior and the solar dynamo at the base of the convective zone, can probably produce variations in the total irradiance. Or, at least, be correlated to these variations in association with the changing emission of bright faculae and the magnetic network (Damé et al., 2000).

Since 1981 the Observatório Nacional/MCT (ON) group makes daily solar observations, in particular, since 1997, aiming to record and study the diameter variations (Jilinski et al., 1998). In 2002 an international network (Réseau de Suivi au Sol du Rayon Solaire - R2S3) was formed to combine the similar work pursued in five countries worldwide (Andrei et al., 2002). In 2006 the ON associated to the SCOSTEP/CAWSES project for the investigation of Space Weather and the Earth-Sun System issues (Andrei et al., 2006). The goal of the work done at $\mathrm{ON}$ is therefore multiple: to record lengthy, coherent series of digitized solar diameter observations; to use this series and those from the R2S3 and other groups to investigate the physics of the photosphere and convection zone; to contribute to the understanding of the Earth-Sun relationship as driven by the heliosphere, with emphasis on short term, geophysics local effects; and finally to upgrade and develop data acquisition and treatment methods and apparatus.

\section{Solar diameter variations}

$\mathrm{ON}$ and R2S3 measures have been consistently obtaining variations of the observed diameter on the order of $100 \mathrm{mas} / \mathrm{solar}$ cycle. Several past and current independent experiments from different groups find (at least) likewise. However, from gravitational energy $(\mathbf{E})$ variation considerations only, a radius $(\mathbf{R})$ variation gives rise to

$$
\Delta E=\Delta R \times\left(3 G M^{2} / 5 R^{2}\right),
$$

where $\mathbf{G}$ is the gravitation constant and $\mathbf{M}$ is the solar mass.

Therefore, to vary the Sun stellar radius by $1 \exp (-4)$ along the $11 \mathrm{y}$ solar cycle, the required energy variation is about 150 times the total solar output (Durney, 1972). Yet, if just the irradiating surface is considered, and assuming that the flux per unit area is kept, a radius variation gives rise to

$$
\Delta I=\Delta R \times(2 / R)
$$

That is, the observed $0.1 \%$ radius variation would explain half of the irradiance $\mathbf{I}$ variation along the solar cycle. Now, if the radius variation is assumed confined to the convective zone (for which in turn an adiabatic regimen is granted), the same $0.1 \%$ variation becomes inexpensive in gravitational energy terms ( $0.01 \%$ in the solar cycle). At the same time, a variation on the convective zone height scale would be quite effective to produce a change of the radius defined by hydrostatic equilibrium, at no expense of gravitational energy and very little change of irradiance.

$$
d I \propto d \rho=d h_{0} \times \rho\left(\exp \left[h / h_{0}^{2}\right]\right),
$$

where $\rho$ is the density and $\mathbf{h}_{\mathbf{0}}$ is the height scale. 
Heliosismology holds that "Results from f-mode oscillations that the solar radius only changes by about $1 \mathrm{~km} /$ year do not preclude the less-sensitive efforts to measure variations of the solar radius at the photosphere by limb observations since the latter are likely to be much larger than the former." (Sofia et al., 2006). So, prevented gravitational or irradiance catastrophes by keeping the physics confined to the convection zone, or better to the photosphere, the massive vault of observations reporting radius variations can be exploited just as the experimental evidence they are.

Some hypothesis to explain the diameter variations have been put forward: localized variations depending of heliolatitude (in special by the equatorial bulge, by the boundary of the royal zone, and at $60^{\circ}$ heliolatitude) - following the magnetic network evolution along the cycle; shear in the differential rotation in the convective zone; self-refraction in the solar atmosphere.

On the other hand, disturbances of the troposphere were for quite a while used as an alternative explanation for the observed variations. Quantitative results were obtained for non-linear quasi-zenith distance dependencies in the case of non-varying prism astrolabes, while an analytic description relates the coherency window (Fried's parameter) and the placement of the inflection point defining the solar limb effective stop. The episode analysis and the compound R2S3 series, bringing together multi-site results from widely separate stations, enforce limits on the effectiveness of those mechanisms (Andrei et al., 2004). Either way, they before all produce strong yearly signals that are not verified, and if prevalent should smear the multi-site signal.

More recently, attention was drawn to the stratosphere modulation with the solar cycle. It would imprint both a mark on multi-site observations (in special from alike instruments, leading to alike biases), due to the effective horizontal mixing, and at the same time as a solar activity related signature. However, it should be considered that the QBO and the yearly stratosphere variations are stronger than the 11 years one, respectively by factors of 2 and 10. On the contrary, for the R2S3 series the 11 year signal is much more evident than one or two year periods. In support to the amplitudes mismatch, it can be also remarked that the 11 year stratospheric modulation befalls on the upward structure, while the solar astrolabe observations are, at least to first order, insensitive to small amplitude refraction and focusing variations.

Combining the self-refraction and height scale variation principles, another mechanism can be postulated. The observed solar radius is just the level beyond which the optical depth reaches unity $(\tau=1)$. The optical depth can be expressed by

$$
\tau=\kappa \rho \times(K T / m g),
$$

where $\kappa$ is the opacity, $\mathbf{K}$ is the Boltzmann's constant, $\mathbf{T}$ is the temperature, $\mathbf{m}$ is the molecular mass, and $\mathbf{g}$ is the gravitational acceleration.

Thus, the optical depth can vary and in so making vary the observed radius in different ways. Either of them will leave a signature on the appearance of the photosphere.

Though granulation forms the very face of sun's photosphere, there are no long term registers of it. Observational and computational hardships to define and follow such highly variable face have so far prevented the realization of those registers (but see Roudier \& Reardon, 1998) even much they are useful for solar physicists. However, in recent years a large, coherent body of white light images became available (Hirzberger et al., 1997). The resolution of the images is at the level of 1 arcsec, thus enabling to perceive granulation structures and individual grains. We retrieved the full solar disk white light images from the Big Bear Solar Observatory (BBSO), at California/USA, covering the raising, peaking, and pos-peak periods of the solar cycle 23 . 
Boscardin et al. (2009) present the results obtained. In conclusion, it is found that the grains sizes are the largest by the solar maximum, in excellent agreement with the maximum of the measured diameter. The grains brightness, on the contrary, is minimum at the solar maximum, and again an excellent agreement is verified with the maximum of the measured diameter. Accordingly, the granulation variation would change the photosphere density, mixing, and eventually its height scale, therefore providing a mechanism to vary the optical depth, and hence the measured diameter.

At the ON $\left(\Phi=-22^{\circ} 53^{\prime} 42^{\prime \prime}, \Lambda=+2 \mathrm{~h} 52 \mathrm{~m} 53 \mathrm{~s} .5, \mathrm{~h}=33 \mathrm{~m}\right)$ the series of solar semidiameter measurements started in 1997. The original Danjon Astrolabe was specially redesigned for this type of observations. The most important features were the installation of a variable angle front prism enabling the continuous observations between the zenith distance of $26^{\circ}$ and $56^{\circ}$, the concurrent installation of a moving density filter, and the installation of a CCD camera, which allowed the observations to become fully freed of personal equations (Jilinski et al., 1999).

The solar semi-diameter series treated here was observed with the CCD Astrolabe from March 2nd 1998 to November 27th 2003. It comprises more than 18,000 observations, with mean internal error of 0 ".20 and standard deviation of $0 . " 59$. The observations are made daily, to an average of 20 observations (actually observing days considered), and even distribution of the measurements all year around. A major gap is verified on the series from Sept. 21th to Dec. 19th, 2001, due to apparatus problems. The observations are taken on before and after meridian sessions. As a rule, there is no significant difference between the measurements from the two sessions. The heliolatitude coverage covers the whole solar figure in a semi-annual cycle (Boscardin, 2004).

From an analysis on heliolatitude bins, the solar oblateness can be derived. A conservative approach was chosen, and an ellipsoid of revolution was directly adjusted to the data, in view of the errors involved in the measurements as well as possible systematic effects due to brightness variations on the observed limb. All data considered, we obtain an equatorial radius ( $\mathbf{R e})$ equal to $959.113 \pm 0.007$ and a polar radius $(\mathbf{R p})$ equal to $959.100 \pm 0.011$. In order to improve on the precision of the determination, and taking advantage of the large number of independent measurements and their normal distribution, the series was sampled by progressively removing the more discrepant points (relatively to an ellipsoid adjustment). We took steps of 0.1 units of standard deviation, removing from $2 \sigma$ up to $3 \sigma$. In the first case, the strictest, 7556 measurements are used, while in the more relaxed case 9047 measurements are used. By this method the robustness of the solution was tested by bootstrapping. The final equatorial and polar radii are given by the average of the values at each step. The final errors are calculated by the root mean square of the squared error at each step plus the covariance of adjacent steps. The final value obtained for the oblateness is $13 \pm 4$ mas, corresponding to slightly smaller radii $\mathbf{R e}=959.110 \pm 0.002$ and $\mathbf{R p}=959.097 \pm 0.003$. Adopting the component of the oblateness due to surface rotation alone as $\delta \mathbf{r}=7.8$ mas, the gravitational quadrupole moment of the Sun is obtained as $\mathbf{J}_{2}=3.61 \pm 2.90 \times 10^{-6}$ (Reis Neto et al., 2003).

The data file here concerned comprises the rise, crest and beginning of the subduing of the solar cycle 23. Combined to the large quantity and even spreading of the observations, this offers the conditions to statistically compare the observed solar semi-diameter variations against estimators of the solar activity, which could also be expressed as continuous daily series. The estimators were the sunspot count number and its proxy, the $10.7 \mathrm{~cm}$ radio flux, both sensing the photosphere state; the total solar irradiance and the strength of the integrated solar magnetic field, sensing directly the solar cycle age, and finally the flare index to assess the major solar outbursts. All estimator data were retrieved from the National Geophysical Data Center - NGDC. The hypothesis that the variation of the 
Table 1. Maximum of the Pearson Linear Correlation between the Time Variations of the Semi-Di ameter and Estimators of the Solar Activity (1998-2003).

\begin{tabular}{cccccc}
\hline Pair & SD-SS $^{1}$ & SD-FL $^{2}$ & SD-RF $^{3}$ & SD-IR $^{4}$ & SD-MF $^{5}$ \\
\hline Correlation & 0.80 & 0.66 & 0.88 & 0.78 & 0.62 \\
\hline Delay (day) & 90 & 100 & 90 & 180 & 120 \\
\hline
\end{tabular}

Notes:

${ }^{1}$ Measured Semi-diameter against sunspot count variations.

${ }^{2}$ Measured Semi-diameter against flare index.

${ }^{3}$ Measured Semi-diameter against $10.7 \mathrm{~cm}$ emission variations.

${ }_{5}^{4}$ Measured Semi-diameter against total irradiance variations.

${ }^{5}$ Measured Semi-diameter against compound magnetic field variations.

solar semi-diameter could be linked to the solar activity was checked by calculating the correlations between the solar semi-diameter series against each one of those estimators. Afterwards, the correlations were re-calculated, taking pairs of correlated series and allowing variable time delays between them (Boscardin et al., 2009). This may points to interconnected phenomena, either with some time delay between them, or even a causal relationship.

In particular, when the periods where had occurred peaks of solar activities are removed from the solar semi-diameter and from the total solar irradiance series, the time delay for the largest correlations between them changes prominently. Taking the complete, no-removal series, the semi-diameter versus irradiance correlation shows two maxima, one close to zero and one close to -380 days. The periods of most intense solar activity are between the Julian modified dates 1709.0 and 1919.0 and between 2213.0 and 2378.0. They are removed from the series. In this case the maximum close to zero disappears. This suggests two modes of response of the semi diameter relatively to the solar irradiance. Along the solar activity cycle the semi-diameter variation trails behind the cycle. However when peaks of activity occur, it also occurs a rapid variation on the measured semi diameter. In these cases the semi diameter variation actually acts as a predictor of intense solar activity.

\section{Conclusions and Perspectives}

The physics of the photosphere is important for the understanding of the solar flux, from the convection zone to the violent outbursts that emerge from the solar outer layers. It is also all relevant to understand the space weather and relationships to the Earth's magnetosphere, ionosphere, and atmosphere.

The ON series enables to obtain a broader description of the photosphere physical processes, including the relationships between the variations of the diameter and of the main estimators of the solar activity. The correlations between the variations along the solar cycle attain 0.78 for the pair of diameter and total solar irradiance, 0.80 for the pair of diameter and sunspot number, and 0.88 for the pair of diameter and $10.7 \mathrm{~cm}$ radio flux.

Also, from the analysis grouping the results in heliolatitude bins, the solar oblateness is derived to a precision of -3 dex.

\section{Acknowledgements}

The authors acknowledge partial financial support from the Brazilian Science Funding Agencies FAPERJ, CNPq, and FINEP. We also acknowledge the use of solar data from 
the BBSO and from the NGDC. We register the fruitful relationship between the national institutions belonging in the R2S3 solar network and in the SCOSTEP/CAWSES project.

\section{References}

Andrei, A. H., Penna, J. L., Reis Neto, E., D‘Ávila, V. A., de Almeida, W. G., \& Jilinski, E. G. 2002, What are the astrolabe observations of the Sun measuring?, First Annual Meeting of the R2S3, Calern, France.

Andrei, A. H., Boscardin, S. C., Chollet, F., Delmas, C., Golbasi, O., Jilinski, E. G., Kili, H., Laclare, F., Morand, F., Penna, J. L., \& Reis Neto, E. 2004, Comparison of CCD astrolabe multi-site solar diameter observations, Astronomy \& Astrophysics, 427, 717-723.

Andrei, A. H., Boscardin, S. C., Reis Neto, E., Penna, J. L., \& D‘Ávila, V. A. 2006, Variations of the solar radius in solar cycle 23, SCOSTEP-CAWSES Meeting, 1, 43-44.

Boscardin, S. C. 2004, Diameter observations analysis in the solar activity context, MSc Thesis, UFRJ, Observatorio do Valongo.

Boscardin, S. C., Reis Neto, E., Penna, J. L., Papa, A. R. R., Andrei, A. H., \& D‘Ávila, V. A. 2009, Observed variations of the solar photospheric diameter, 2009, IAU Symposium 264, Solar and Stellar Variability - Impact on Earth and Planets.

Damé, L., Brun, J.-F., Cugnet, D., Derrien, M., Leroy, C., Meftah, M., Meissonnier, M., \& Porteneuve, J. 2000, A solar diameter metrology measurement: the PICCARD microsatellite program, ICSO 2000.

Durney, B. R. \& Roxburgh, I. W. 1971, Inhomogeneous convection and the equatorial acceleration of the Sun, Solar Physics, 16, 3-11.

Hirzberger, J., Vazquez, M., Bonet, J. A., Hansmeier, A., \& Sobotka, M. 1997, The series of solar granulation inages. I. Differences between small and large granules in quiet regions, The Astrophysical Journal, 480, 406-419.

Jilinski, E. G., Puliaev, S. P., Penna, J. K., Andrei, A. H., Sinceac, V., Chollet, F., \& Delmas, C. 1998, Solar diameter observations with the astrolabe at Observatório Nacional Rio de Janeiro, Astronomy \& Astrophysics Supplement Series, 130, 317-321.

Mursula, K. 2005, Sun and Heliosphere, University of Oulu.

Reis Neto, E., Andrei, A. H., Pennal, J. L., Jilinski, E. G., \& Puliaev, S. P. 2003, Observed variations of the solar diameter in 1998-2000, Solar Physics, 212, 7-16.

Roudier, Th. \& Reardon, K. 1998, Variations of the solar granulation over the cycle: previous results and future observations, Synoptic Solar Physics, 140, 455-466.

Sofia, S., Basu, S., Demarque, P., Linghuai, L., \& Thuiller, G. 2006, The nonhomologous nature of solar diameter variations, Astrophysical Journal, 632, 439. 